\title{
DOROTHY KenNy
}

\section{Die Übersetzung von usuellen und nicht usuellen Wortverbindungen vom Deutschen ins Englische}

\author{
Eine korpusgestützte Untersuchung
}

\begin{abstract}
In dieser Studie wird die Übersetzung von Kollokationen untersucht, die das Lemma AUGE und verschiedene Verben des Öffnens und des Schließens verbinden. Als Datenquelle für die Studie dient GEPCOLT (German-English Parallel Corpus of Literary Texts). Die Relevanz dieser Untersuchung für die Übersetzungswissenschaft und das Übersetzen selbst wird im zweiten Teil diskutiert. Es wird herausgestellt, wie Standardübersetzungen Fälle von Delexikalisierung in der Ausgangssprache veranschaulichen können, und gezeigt, dass verschiedene formale Realisierungen derselben semantischen Kollokation mehr oder weniger stabile Übersetzungen produzieren können. Zum Schluß wird dargelegt, wie nützlich diese Art der Analyse für Stilstudien sein kann, insofern als die konsequente Verwendung eines bevorzugten Wortes ein Element des individuellen Stils einer Übersetzerin/eines Übersetzers ist.
\end{abstract}

\section{Einleitung}

In der vorliegenden Studie wird die Übersetzung von deutschen Kollokationen untersucht, die das Lemma AUGE und verschiedene Verben des Öffnens und des Schließens verbinden. Als Datenquelle für die Studie dient GEPCOLT (Kenny 2001), ein Parallelkorpus, das aus literarischen deutschen Texten und deren Übersetzungen ins Englische besteht. Im ersten Teil der Studie wird das Korpus vorgestellt und begründet, warum der Schwerpunkt auf Kollokationen mit dem Lemma AUGE gelegt wird. Die Analyse der betreffenden Kollokationen auf Deutsch und Englisch folgt im zweiten Teil. Die Studie endet mit einigen Bemerkungen über die Bedeutung der Ergebnisse dieser Analyse für die Übersetzungswissenschaft und ihre Nützlichkeit für zukünftige Studien.

Zunächst soll erläutert werden, warum es sinnvoll ist, Parallelkorpora zu untersuchen. Auf dem Gebiet der Übersetzungswissenschaft spielt die Untersuchung des sprachlichen Verhaltens von ÜbersetzerInnen eine zentrale Rolle (S. dazu Kenny 2001, S. 57-69). Parallelkorpora bieten die Möglichkeit, dieses sprachliche Verhalten, das nur zum Teil von den Ausgangstexten bestimmt wird, systematisch und unter verschiedenen Gesichtspunkten zu untersuchen. Darüber hinaus gibt es die Möglichkeit der praktischen Anwendung von Parallelkorpora, z. B. in der Lexikografie und der computergestütz- 
ten Übersetzung. Wollen wir die zweisprachige Lexikografie auf eine empirische Basis stellen, wie es bei vielen einsprachigen Wörterbüchern schon üblich ist, müssen wir Übersetzungseinheiten in Parallelkorpora identifizieren. Im Bereich computergestützter Übersetzungen sind Translation Memories von Interesse. Sie speichern bereits übersetzte Ausgangstexte bzw. Sätze in der Ausgangssprache zusammen mit ihren Zieltexten oder Zielsätzen, damit sie auf diese Übersetzungseinheiten zurückgreifen können. Die Nützlichkeit dieser Technologie beruht auf immer größeren Parallelkorpora, deren Übersetzungseinheiten immer wieder recycelt werden können.

Wenn es aber Besonderheiten im sprachlichen Verhalten von ÜbersetzerInnen gibt, wäre es sinnvoll diese zu erkennen, bevor sie in zweisprachigen Wörterbüchern erscheinen bzw. mit Hilfe von Translation Memories verbreitet werden.

\section{GEPCOLT}

Zum Korpus: GEPCOLT ist das englischsprachige Akronym für GermanEnglish Parallel Corpus Of Literary Texts. Das Korpus besteht aus vierzehn deutschen literarischen Texten und deren Übersetzungen ins Englische. Die meisten deutschen Texte stammen aus den 80er und 90er Jahren, die englischen überwiegend aus den 90er Jahren. Die genaue Zusammensetzung des Korpus wird in den Quellen angegeben.

GEPCOLT umfasst insgesamt zwei Millionen Textwörter, d.h. ca. 1 Million Textwörter je Sprache. Das Korpus wurde zwischen 1997 und 1998 für eine Doktorarbeit (Kenny 1999) aufgebaut, und zwar mit dem Ziel, etwas über die heutigen Gewohnheiten des Übersetzens vom Deutschen ins Englische aussagen zu können. Alle Texte wurden mit Genehmigung der InhaberInnen der Urheberrechte in elektronische Form umgewandelt, um die elektronische Bearbeitung der Daten zu ermöglichen. Für die Analyse des Korpus wurden zwei verschiedene Softwareprogramme benutzt. WordSmith Tools (Scott 1997) diente zur Erstellung von Frequency-Listen, d. h. nach der Häufigkeit sortierten Wortlisten, und einsprachigen Konkordanzen. Die zweisprachigen Konkordanzen in den unten angeführten Belegen wurden mit Multiconcord (Woolls 1997) erstellt.

\section{AUGE als Kollokationsbasis}

AUGE wird in den deutschen Texten häufig verwendet. Wie aus Abbildung 1 hervorgeht, kommt die gebräuchlichste Form, Augen im Plural, 1.017 Mal im Korpus vor und steht damit an Stelle 110 der Frequency-Liste. Auge kommt $129 \mathrm{Mal}$ vor, und Auges $13 \mathrm{Mal}$. Die relativ hohe Häufigkeit dieses Lemmas läßt eine Analyse seines Kollokatspotentials (Lehr 1996, S. 39-43) sinnvoll erscheinen. Darüber hinaus haben die im Englischen am häufigsten verwendeten Wörter laut Stubbs (2001) eine starke Tendenz, mit einer beschränkten Gruppe von Kollokaten aufzutreten. Wenn diese Behauptung so- 
wohl auf das Deutsche als auch auf das Englische zutrifft, dann sollte Augen als häufig vorkommendes Wort interessante Möglichkeiten für die Analyse wiederholter Kollokationen bieten. Darüber hinaus sollten vor dem Hintergrund wiederholter, üblicher Kollokationen auch unübliche Kollokationen betrachtet werden.

\begin{tabular}{|c|c|c|}
\hline Type & Rank & $\begin{array}{c}\text { Häufigkeit in } \\
\text { GEPCOLT }\end{array}$ \\
\hline Augen & 110 & 1.017 \\
\hline Auge & 689 & 129 \\
\hline Auges & 5.138 & 13 \\
\hline & & 1.159 \\
\hline
\end{tabular}

Abb. 1: Häufigkeit des Lemmas AUGE in den deutschen Texten in GEPCOLT.

Aus Kenny (2001) ließ sich ableiten, dass in den deutschen Texten, verglichen mit den englischen Texten, eine größere Vielfalt an Verben benutzt wird, um das Öffnen der Augen zu beschreiben. Das heißt, es schien in diesem beschränkten semantischen Bereich einen bemerkenswerten Unterschied zwischen Deutsch und Englisch zu geben, der einer weiteren Analyse wert war. Im Laufe der vorliegenden Studie zeichnete sich außerdem ab, dass Kollokationen von Augen mit Verben des Öffnens interessante Daten für eine Untersuchung über Delexikalisierung liefern könnten. Eine Analyse solcher Kollokationen schien daher gerechtfertigt. Die anschließende Untersuchung von Kollokationen mit Verben des Schließens schien der Vollständigkeit halber angemessen.

\section{Datenanalyse}

68 von den 1.017 Fällen der Pluralform Augen im Korpus kollokieren mit einem Verb des Öffnens. Die entsprechenden Verben und die Häufigkeit ihres Vorkommens mit Augen werden in Abbildung 2 aufgeführt.'

Das bei weitem häufigste Verb dieses semantischen Bereichs, das mit $A u$ gen auftritt, ist unüberraschenderweise das Verb ÖFFNEN. ÖFFNEN gilt als Grundvokabel der deutschen Sprache, insofern als es ein sehr gebräuchliches Verb ist und weitgehend in Definitionen von verwandten Verben, wie z. B. AufSPERREN, AUFREIBEN usw. benutzt wird. Wie Abbildung 3 zeigt, tritt ÖFFNEN häufig als Vollverb des Satzes mit Augen auf, das heißt als finites

' Es soll erwähnt werden, dass es hier um Verben geht, die den Vorgang des Augenöffnens betonen können. Es gibt natürlich auch Verben und andere sprachliche Mittel im Korpus, die den Zustand von offenen Augen betonen, z. B. AUFSTEHEN, OFFENSTEHEN und OFFEnHALTEN. Diese werden aber nicht in die vorliegende Studie einbezogen. 


\begin{tabular}{|c|c|}
\hline Lemma & Häufigkeit mit Augen \\
\hline ÖFFNEN & 32 \\
\hline AUFREIßEN & 18 \\
\hline AUFSCHLAGEN & 5 \\
\hline AUFMACHEN & 4 \\
\hline AUFSPERREN & 3 \\
\hline AUFKLAPPEN & 2 \\
\hline AUFZWÄNGEN & 2 \\
\hline AUFGEHEN & 1 \\
\hline AUFKRIEGEN & 1 \\
\hline & 68 \\
\hline
\end{tabular}

Abb. 2: Häufigkeit der Verben des Öffnens mit Augen.

Verb oder als Prädikat im hallidayschen Sinne. Es wird in diesen Fällen erwartungsgemäß fast immer mit dem englischen OPEN übersetzt.

\begin{tabular}{|c|c|c|}
\hline ÖFFNEN & Übersetzung & Häufigkeit \\
\hline Vollverb & OPEN & 25 \\
\hline Vollverb & SNAP OPEN & 1 \\
\hline Vollverb & $\varnothing$ & 1 \\
\hline andere Funktionen & $\begin{array}{c}\text { wide open, newly-opened, } \\
\text { half-opened, with slit eyes, } \\
\text { wide-eyed }\end{array}$ & 5 \\
\hline & & 32 \\
\hline
\end{tabular}

Abb. 3: Übersetzungen von ÖFFNEN in Kollokationen mit Augen.

In einem einzigen Fall benutzt der Übersetzer das Lemma SNAP OPEN, um ÖFFNEN zu übersetzen (Beleg 1). ${ }^{2}$ Hier könnte man vermuten, dass der Übersetzer das schon erwähnte Drama der Szene hervorheben oder Abwechslung in seinen Text bringen möchte.

1a „Etwas weniger klug und nicht ganz so dramatisch“, flüsterte Horgan. Augustin öffnete die Augen. „Aber ich bin, wie ich bin“, erwiderte er. [kirchhof.de]

\footnotetext{
${ }^{2}$ Die relevanten Stellen sind in den Belegen kursiv hervorgehoben.
} 
Ib Come down off your high horse, my son, „Horgan whispered.“ „Try to be a little less melodramatic.“ Augustin's eyes snapped open. „But that's the way I am,“ he said, ,all heart.“ [kirchhof.en]

In einem zweiten Fall (Beleg 2) wird ein ganzer Satz ,und dabei öffnete er die Augen" nicht übersetzt.

2a So schnell könnte ich nicht mehr laufen, mußte Vogtmann denken, und dabei öffnete er die Augen und wußte sofort den Namen des Hotels und des Orts und sogar das Datum, und auch alles andere kam sofort zurück: [weller.de]

2b I couldn't run that fast any more, Vogtmann couldn't help thinking, $\varnothing$ and immediately he knew the name of the hotel and the place and even the date, and everything else came back immediately: [weller.en]

Hat das Lemma ÖFFNEN nicht die Funktion des finiten Verbs oder Prädikats im Satz, sind die Übersetzungen vielfältiger. In den Belegen 3a bis 7a wird ÖFFNEN als Ergänzung des Verbs SEIN oder zur näheren Bestimmung eines Substantivs (normalerweise in einer Präpositionalphrase) benutzt. Trotz der wenigen Daten können wir vorläufig sagen, dass in diesen Fällen freier übersetzt wird.

3a Sie legen die Flügel an, und aus ihren Leibern schießen die Kinder, in deren neu geöffnete Augen die Väter ihre Blitze schleudern. [jelinek1.de]

$3 b$ They fold in their wings, and from their bodies the children shoot forth, and the fathers zap their flashing lightning into the kiddies' newly-opened eyes. [jelinek $1 . e n]$

4a L sah mich an, mit weit geöffneten Augen: Ich liebe dich. [wodin.de]

4b L looked at me wide-eyed: „I love you.“ [wodin.en]

5a Ihre Augen waren weit geöffnet und sahen ihn an. [weller.de]

$5 \mathrm{~b}$ Her eyes were wide open, looking at him. [weller.en]

6a Lange scheint sie, die Augen schmal geöffnet, noch auf unsere Wunde zu schauen, [hofmann.de]

$6 \mathrm{~b}$ For a long time she seems to be looking with slit eyes at our wound still, [hofmann.en]

7a ihr Kopf mit den halbgeöffneten Augen, in denen das Weiß der verdrehten Augäpfel schimmerte, war zur Seite gerollt ... [weller.de]

$7 \mathrm{~b}$ her head with the half-opened eyes in which the white of the rolled eyeballs gleamed had rolled to the side ... [weller.en]

Bezüglich der anderen Verben des Öffnens geht aus Abbildung 2 hervor, dass AUFREIBEN das zweithäufigste Verb ist, das im Korpus mit Augen vorkommt. Die Übersetzungen für dieses Verb werden in Abbildung 4 aufgeführt.

Die gebräuchlichste Übersetzung des Lemmas AUFREIBEN ist die Standardübersetzung OPEN WIDE, wie sie in zweisprachigen Wörterbüchern, z.B. im Collins Pons vorgeschlagen wird. OPEN wird zweimal allein benutzt und die 


\begin{tabular}{|c|c|c|}
\hline AUFREIBEN (Funktion) & Übersetzung & Häufigkeit \\
\hline Vollverb & OPEN WIDE & 5 \\
\hline & OPEN & 2 \\
\hline & FORCE OPEN (8b) & 1 \\
\hline & STARE, wide-eyed (15b) & 1 \\
\hline Ergänzung des Verbs & Go wide as saucers (16b) & 1 \\
\hline SEIN & BE wide open & 2 \\
\hline Bestimmungswort in & BE half open & 1 \\
\hline einer Nominalgruppe & gawping wide-eyed at all & 1 \\
& $\begin{array}{c}\text { that was going on, } \\
\text { wide open, open-eyed, }\end{array}$ & 1 \\
& eyes popping out of her \\
& head & 1 \\
\hline & & 18 \\
\hline
\end{tabular}

Abb. 4: Übersetzungen von AUFREIBEN in Kollokationen mit Augen.

Übersetzerinnen von Biermanns Roman verwenden einmal FORCE OPEN, um AUFREIBEN zu übersetzen. Wie jedoch in den Belegen $8 \mathrm{~b}$ bis $13 \mathrm{~b}$ zu sehen ist, bevorzugen sie die Standardübersetzung OPEN WIDE.

Der Übersetzer von Erich Loests Völkerschlachtdenkmal, Ian Mitchell, weicht von dieser Standardübersetzung in den Belegen 15b und 16b ab. Für ihn verlangen die ausgangssprachlichen Ausdrücke emphatischere Übersetzungen. Diese könnten ein Ausgangspunkt für weitere Analysen über den Stil von ÜbersetzerInnen sein.

8a Er riß die Augen wieder auf und starrte in fünf Fratzen, die vor Gelächter und Verachtung zu bersten schienen. [bier.de]

$8 \mathrm{~b} \mathrm{He}$ forced his eyes open again and looked into five faces which seemed to be bursting with laughter and contempt. [bier.en]

9a Er riß die Augen wieder auf, klatschte die Zeitung noch einmal gegen die Wand und ließ sie fallen [bier.de]

$9 \mathrm{~b}$ He opened his eyes wide again, smacked the newspaper against the wall once more and dropped it. [bier.en]

10a Lietze riß die Augen auf, [bier.de]

$10 \mathrm{~b}$ Lietze opened her eyes wide, [bier.en]

11a Sie schoß im Bett hoch und versuchte, die Augen aufzureißen. [bier.de]

$11 \mathrm{~b}$ She shot up in bed and tried to open her eyes. [bier.en]

12a Der junge Mann, der nackt hinter dem Türspalt stand, riß jetzt doch die schlafverklebten Augen auf. [bier.de] 
$12 \mathrm{~b}$ The young man standing naked behind the half open door now opened his sleepy eyes wide. „Tak - ash. [bier.en]

13a Als er zwischen zwei Schneuzern die Augen aufriß und das Objekt seiner observierenden Begierde ins Visier nahm, ging die Haustür auf, und ein untersetzter Mann trat auf die Straße. [bier.de]

$13 \mathrm{~b}$ As he opened his eyes wide between sneezes and got a clear look at the object of his surveillance, the front door of the building opened and a stocky man stepped into the street. [bier.en]

14a Er sah die Soldatenkolosse über Säuglinge steigen, Säuglinge, die auf dem Bordstein lagen und ihre Augen aufrissen, sobald die Menschentürme über ihnen erschienen; [kirchhof.de]

14b He fled into the street and saw colossal soldiers stepping over bundles - infants that lay on the pavement and opened their eyes when the human towers loomed over them. [kirchhof.en]

15a Wir standen starr, als Kommandos erschollen, als wären auch wir betroffen, rissen die Augen auf, als sich die Männer, Felix Linden unter ihnen, in eine Reihe schoben und die Köpfe ruckten, ... [loest.de]

$15 \mathrm{~b}$ We stood to attention as commands rang out, as if they were aimed at us, too, and we stared, wide-eyed, as the men, Felix Linden among them, formed into a line and snapped their heads back, [loest.en]

16a Meiner Marianne erzählte ich mittags, was ich mit angesehen hatte, sie riß die Augen auf. [loest.de]

16b Over lunch, I told my Marianne all about what I had seen, and her eyes went wide as saucers. [loest.en]

17a Die Hühner ... reißen ihre Augen weit auf und rühren sich nicht, weil Hühner sich beim Eierlegen nicht rühren können. [wodin.de]

$17 \mathrm{~b}$ The hens ... open their eyes very wide and don't move because hens can't move while they're laying. [wodin.en]

18a Die Augen halb aufgerissen. Oder halb zugezwängt. Von zu schweren Lidern. [bier.de]

$18 \mathrm{~b}$ Her eyes are half open or half shut. The lids too heavy. [bier.en]

19a ... die Augen waren aufgerissen, der Hinterkopf fehlte und klebte hinter ihm an der Wand als eine blutige Aureole. [weller.de]

$19 \mathrm{~b}$ His eyes were wide open, and the back of his head was missing, stuck to the wall behind him like a bloody halo. [weller.en]

Soweit scheint die Variabilität der Übersetzungen von AUFREIBEN vom Übersetzer bzw. von der Übersetzerin abzuhängen. Betrachten wir jedoch Fälle, in denen das Lemma AUFREIßEN als Bestimmungswort in einer Nominalgruppe vorkommt, finden wir unterschiedliche Übersetzungen (Belege 20a bis 25a).

20a Sie hatten aufgerissene Augen und rote, emporstehende Haare. [zuernl.de] 
$20 \mathrm{~b}$ They had gawping eyes and red hair which stood on end. [zuern $1 . e n]$

21a Einige von ihnen setzten einfach ihre Kinder im Heim ab, für ein paar Tage, die standen dann herum, mit weit aufgerissenen Augen, in besonders gepflegten Kleidern. [gold.de]

$21 \mathrm{~b}$ Some simply left their children at the home for a few days, they would stand around, gazing wide-eyed at all that was going on, wearing particularly smart clothes. [gold.en]

22a Es war ein Mann mit weitaufgerissenen Augen, vermutlich ein abgestürzter Bergsteiger, der einen Pickel in der Rechten hielt. [roth2.de]

$22 \mathrm{~b}$ A man with wide open eyes, presumably a mountaineer who had fallen to his death, holding an ice pick in his right hand. [roth2.en]

23a Sie kam mit aufgerissenen Augen von einem Schrecken zurück, [weller.de]

$23 \mathrm{~b}$ She was returning open-eyed from a horror ... [weller.en]

24a ... und ich sehe, was ich sehen will, den Ausdruck des tödlichen, über alles hinausgehenden Grauens in Ljudas Gesicht, einen Augenblick erstarrt, mit weit aufgerissenen Augen; [wodin.de]

$24 \mathrm{~b} . .$. and I see what I wanted to see: a look of deathly, all-transcending terror on Lyuda's face, frozen for an instant, eyes popping out of her head. [wodin.en]

25a durch die Mauer des Grauens, das in seinen weit aufgerissenen Augen steht, [wodin.de]

$25 \mathrm{~b}$ I felt sure I had only to call his name, convinced that the sound of my voice would penetrate the wall of dread that loomed in his wide open eyes. [wodin.en]

Diese vorläufigen Ergebnisse sowie die angeführten Belege für das Lemma ÖFFNEN könnten auf eine eventuell interessante Forschungsfrage hindeuten: Produzieren verschiedene formale Realisierungen derselben semantischen Kollokation meistens dieselben Übersetzungen oder nicht?

\section{Delexikalisierung}

Das Lemma Aufreiben bietet auch die Gelegenheit, das Phänomen der Delexikalisierung zu betrachten. Delexikalisierung heißt, dass ein Wort in einer Mehrworteinheit seinen unabhängigen Sinn verliert. Dieses Wort kann zwar eine wichtige grammatische Funktion haben, es kommt aber semantisch leer vor. Ein Beispiel der deutschen Sprache ist das Verb TREFFEN in der Kollokation eine Entscheidung treffen. Laut Sinclair (1992) sind einige Adjektive, die in häufig vorkommenden Kollokationen auftreten, z.B. ,general" in ,general public" oder ,general trend", zum Teil delexikalisiert. Solche Adjektive dienen nicht mehr dazu, eine Untergruppe des betreffenden Substantivs zu bezeichnen. Sie betonen oder wiederholen vielmehr den Inhalt des Substantivs und weisen dadurch eine gewisse semantische Überflüssigkeit auf, obwohl sie pragmatisch wichtig sind. Gunter Lorenz (1999) hat 
eine ähnlich interessante Studie über delexikalisierte Adverbien durchgeführt.

Für das Lemma AUFREIBEN geben einsprachige Wörterbücher wie der Wahrig (Wahrig 2000) den Sinn von weit öffnen wie folgt an:

auf|reißen $1<\mathrm{V} .195>$ durch Reißen öffnen (Brief); ...<fig.> schnell $u$. weit öffnen (Augen, Mund); (Wahrig 2000; Hervorhebung im Original)

Im Duden (1993) wird der Sinn von weit öffnen mit der Umgangsprache verbunden:

auf|rei|ßen: <st. V.> 1. durch [Zer]reißen [der Umhüllung] öffnen $<$ hat $>$ : einen Brief, eine Zigarettenpackung a.; 2. schnell, ruckartig öffnen $<$ hat $>$ : die Tür a.; ... den Mund, die Augen a. (ugs.; vor Schreck, Staunen o. ä. weit öffnen). (Duden 1993; Hervorhebung im Original)

Diese Definition ist in Langenscheidts Wörterbuch Deutsch als Fremdsprache (Götz/Haensch/Wellmann 1997) impliziert. AUFSPERREN wird als Synonym für AUFREIBEN angegeben, und AUFSPERREN wird als „etwas weit öffnen" definiert.

auf(reiß(en Vt (hat) 1 etw. a. etw. mst. durch Zerreißen der Hülle öffnen <en Brief, e-n Beutel a.> 2 etw. a. etw. plötzlich u. schnell öffnen $<$ den Mund, das Fenster, a.> ... (Langenscheidts Großwörterbuch DaF 1997; Hervorhebung im Original)

aufsperren (hat) Vt ... 2 etw. a. gespr; etw. weit öffnen $\approx \sim$ aufreißen (2) $<$ den Mund weit a.> (Langenscheidts Großwörterbuch DaF 1997; Hervorhebung im Original)

Wenn man bedenkt, dass zweisprachige Wörterbücher wie der Collins-Pons (Terrell/Schnorr/Morris/Breitsprecher 1997) AUFREIBEN mit OPEN WIDE übersetzen, dann scheint das Lemma AUFREIBEN den Sinn von weit zu enthalten, sowohl bezüglich des Deutschen als auch aus der Perspektive der Fremdsprache. Man könnte daher sagen, dass weit in den Belegen 17a, 21a, 22a, 24a, und 25a delexikalisiert ist und als Verstärkungswort benutzt wird.

Es gibt aber ÜbersetzerInnen, die diese Funktion offensichtlich übersehen. Kommt „weit aufreißen“ in einem Text vor, übersetzen sie es mit einem zusätzlichen Verstärkungswort (Beleg 17b).

17a Die Hühner ... reißen ihre Augen weit auf und rühren sich nicht, weil Hühner sich beim Eierlegen nicht rühren können. [wodin.de]

$17 \mathrm{~b}$ The hens ... open their eyes very wide and don't move because hens can't move while they're laying. [wodin.en]

Wie wir gesehen haben, können Standardübersetzungen dazu dienen, Delexikalisierung in der Ausgangssprache zu verdeutlichen. Damit kann man wiederum längere Sinneinheiten in der Ausgangssprache identifizieren, denn ein delexikalisiertes Wort sollte nicht isoliert sondern als Teil einer längeren Sinneinheit betrachtet werden. 


\section{Nicht usuelle Wortverbindungen}

Abbildung 5 zeigt die Übersetzungen der anderen Verben des Öffnens, die in GEPCOLT mit Augen kollokieren, z.B. AUfSCHLAGEN, AUFMACHEN, AUFSPERREN Und AUFKLAPPEN.

\begin{tabular}{|c|c|c|}
\hline LEMMA & Übersetzung & Häufigkeit \\
\hline AUFSCHLAGEN & OPEN & 5 \\
\hline AUFMACHEN & OPEN & 4 \\
\hline AUFSPERREN & KEEP OPEN & 1 \\
& STARE & 1 \\
& OPEN WIDE & 1 \\
\hline AUFKLAPPEN & OPEN & 2 \\
\hline AUFZWANNGEN & FORCE OPEN & 2 \\
\hline AUFGEHEN & ROLL & 1 \\
\hline AUFKRIEGEN & OPEN & 1 \\
\hline & & 18 \\
\hline
\end{tabular}

Abb. 5: Übersetzungen anderer Verben des Öffnens in Kollokationen mit Augen.

Hier fällt eine Übersetzung von AUFSPERREN auf (Beleg 26):

26a Der junge Mann wird seine Augen aufsperren gehen, wenn er die neue Frisur sieht. [jelinek1.de]

$26 \mathrm{~b}$ The young man will stare a wide open unlocked stare when he sees the new hairdo. [jelinekl.en]

Der Ausgangssatz, aus dem Roman Lust von Elfriede Jelinek, ist grammatisch und semantisch ungewöhnlich, da es nicht nötig ist, sich von der Stelle zu bewegen, um die Augen aufzusperren. Die Übersetzung ins Englische ist ebenso ungewöhnlich. STARE ist ein intransitives Verb, das als transitives Verb benutzt wird. Das Substantiv STARE wird zusätzlich als Objekt des Verbs benutzt, was einen übertriebenen Sinn von Entschlossenheit und Dauerhaftigkeit schafft. Sätze wie im Beleg 26 veranschaulichen, dass es zu sehr kreativen Übersetzungslösungen kommen kann, wenn ÜbersetzerInnen mit entsprechend kreativen Ausgangstexten zu tun haben.

Abbildung 5 zeigt jedoch, dass ÜbersetzerInnen in den meisten Fällen auf Standardübersetzungen zurückgreifen und dass es in diesem semantischen Bereich im englischen Lexikon weniger Variabilität zu geben scheint als im deutschen Lexikon. 


\section{Verben des Schließens}

SCHLIEßEN ist das gebräuchlichste Verb des Schließens und kommt im Zusammenhang mit Augen am häufigsten im Korpus vor. Des weiteren sind Verben wie ZUMACHEN und ZUDRÜCKEN zu finden. Diese werden am häufigsten mit CLOSE übersetzt (Abb. 6). SHUT wird ebenfalls sehr häufig verwendet und scheint hier ein volles Synonym von CLOSE zu sein. Es fällt auf, dass zwei Drittel der SHuTs von einem Übersetzer (John Brownjohn) stammen. Die Tendenz CLOSE oder SHUT zu benutzen, scheint demnach übersetzerabhängig zu sein. Diese Art von Analyse kann für Stilstudien nützlich sein, wenn man davon ausgeht, dass die konsequente Verwendung eines bevorzugten Wortes ein Element des individuellen Stils eines Übersetzers/einer Übersetzerin ist. Und solche konsequenten Verhaltensmuster lassen sich sehr leicht in korpusbasierten Studien erkennen.

\begin{tabular}{|c|c|}
\hline Lemma & Häufigkeit \\
\hline CLOSE & 85 \\
\hline SHUT & 46 \\
\hline ANDERE VERBEN & 10 \\
\hline & 141 \\
\hline
\end{tabular}

Abb. 6: Übersetzungen von Verben des Schließens.

\section{Schlussbemerkungen}

Dieser Studie liegen Daten relativ geringen Umfangs zugrunde. Es konnte daher in diesem Rahmen nur ein begrenzter Bereich von Kollokationen untersucht werden. Des weiteren wurde kaum berücksichtigt, inwieweit Übersetzungsentscheidungen von den Unterschieden im deutschen und englischen Sprachsystem beeinflusst werden können.

Es konnte jedoch gezeigt werden, dass es trotz wiederholten Gebrauchs von Standardübersetzungen Variabilitäten in den englischen Texten gibt. Diese Variabilität ist teilweise auf unterschiedliche formale Realisierungen derselben semantischen Kollokationen zurückzuführen, könnte teilweise jedoch auch von den Übersetzungskonzepten der unterschiedlichen ÜbersetzerInnen abhängig sein. Die Ergebnisse dieser Analyse unterstützen die in der Übersetzungswissenschaft vertretene Ansicht, dass Übersetzungslösungen trotz der Verwendung von Standardübersetzungen nicht ausschließlich von den Ausgangstexten und sprachsystemischen Unterschieden bestimmt werden, sondern dass sie einen unvermeidbaren subjektiven Einfluß der Übersetzerin/des Übersetzers aufweisen.

Schließlich bleibt zu hoffen, dass die vorgestellten Daten zu weiteren Studien über Delexikalisierung und erweiterte Sinneinheiten und damit erweiterte Übersetzungseinheiten führen werden. 


\section{Quellen (GEPCOLT) [Dateinamen in Klammern]}

Bayer, Konrad (1965): der kopf des vitus bering. Vien. ÖBV-Klett-Cotta Verlagsgesellschaft m.b.H. [bayer.de]

Übersetzung. Billeter, Walter (1994): the head of vitus bering. London. Atlas Press. [bayer.en]

Biermann, Pieke (1990): Violetta. Berlin. Rotbuch Verlag. [bier.de]

Übersetzung. Rieder, Ines/Hannum, Jill (1996): Violetta. London/New York. Serpent's Tail. [bier.en]

Goldschmidt, Georges-Arthur (1991): Die Absonderung. Zürich. Ammann Verlag AG. [gold.de]

Übersetzung. Kirkup, James (1993): Worlds of Difference. London. Quartet Books Ltd. [gold.en]

Hofmann, Gert (1984): Unsere Eroberung. Darmstadt/Neuwied. Hermann Luchterhand Verlag GmbH \& Co KG. [hofmann.de]

Übersetzung. Middleton, Christopher (1987): Our Conquest. Manchester. Carcanet Press Ltd. [hofmann.en]

Jelinek, Elfriede (1980): Die Ausgesperrten. Reinbek bei Hamburg. Rowohlt Verlag $\mathrm{GmbH}$. [jelinek2.de]

Übersetzung. Hulse, Michael (1990): Wonderful, Wonderful Times. London. Serpent's Tail. [jelinek2.en]

Jelinek, Elfriede (1989): Lust. Reinbek bei Hamburg. Rowohlt Verlag GmbH. [jelinek1 .de] Übersetzung. Hulse, Michael (1992): Lust. London/New York. Serpent's Tail. [jelinek 1 .en]

Kirchhoff, Bodo (1990): Infanta. Frankfurt am Main. Suhrkamp Verlag. [kirchhof.de]

Übersetzung. Brownjohn, John (1992): Infanta. London. Harvill. [kirchhof.en]

Loest, Erich (1984): Völkerschlachtdenkmal. Hamburg. Hoffmann und Campe Verlag. [loest.de]

Übersetzung. Mitchell, Ian (1987): The Monument. London. Martin Secker \& Warburg Ltd. [loest.en]

Ransmayr, Christoph (1984): Die Schrecken des Eises und der Finsternis. Vien. Christian Brandstätter Verlag und Edition. [ransmayr.de]

Übersetzung. Woods, John E. (1991): The Terrors Of Ice And Darkness. London. George Weidenfeld and Nicholson Ltd. [ransmayr.en]

Roth, Gerhard (1972): die autobiographie des albert Einstein. Frankfurt am Main. Suhrkamp Verlag. [rothl.de]

Übersetzung. Green, Malcolm (1992): the autobiography of albert Einstein. London. Atlas Press. [rothl .en]

Roth, Gerhard (1986): AmAbgrund. Frankfurt am Main. S. FischerVerlag GmbH.[roth2.de]

Übersetzung. Green, Malcolm (im Druck): On the Brink. London. Atlas Press. [roth2.en]

Wellershoff, Dieter (1983): Der Sieger nimmt alles. Köln. Kiepenheuer und Witsch. [weller.de]

Übersetzung. Knight, Paul (1986): Winner Takes All. Manchester. Carcanet Press Ltd. [weller.en]

Wodin, Natascha (1983): Die gläserne Stadt. Leipzig. Reclam Verlag. [wodin.de]

Übersetzung. Brownjohn, John (1986): The Interpreter. San Diego/New York: Harcourt Brace Jovanovich. [wodin.en]

Zürn,Unica (1977): Der Mann im Jasmin. Frankfurt am Main/Berlin. Verlag Ullstein GmbH.

(Der Mann im Jasmin: Eindrücke aus einer Geisteskrankheit [zuernl de]; Notizen zur letzten (?) Krise [zuern2.de]; Les Jeux à Deux [zuern3.de]; Das Haus der Krankheiten [zuern4.de]) 
Übersetzung. Green, Malcolm (1993): The House of Illnesses. London. Atlas Press. [zuern4.en]; Green, Malcolm (1994): The Man of Jasmine \& Other Texts. London. Atlas Press. (The Man of Jasmine: Impressions from a Mental Illness. [zuern1.en]; Notes on Her Last (?) Crisis [zuern2.en]; Les Jeux à Deux [zuern3.en])

\section{Literatur}

Duden (1993): Das große Wörterbuch der deutschen Sprache in acht Bänden. 2. Auflage. Mannheim/Leipzig/Wien/Zürich: Dudenverlag.

Götz, Dieter/Haensch, Günther/Wellmann, Hans (Hg.)(1997): Langenscheidts Großwörterbuch Deutsch als Fremdsprache. Berlin/München/Wien/Zürich/New York: Langenscheidt.

Kenny, Dorothy (1999): Norms and Creativity: Lexis in Translated Text. Unveröffentlichte Doktorarbeit. Manchester: UMIST.

Kenny, Dorothy (2001): Lexis and Creativity in Translation: A corpus-based study. Manchester: St. Jerome.

Lehr, Andrea (1996): Kollokationen und maschinenlesbare Korpora: ein operationales Analysemodell zum Aufbau lexicalischer Netze. Tübingen: Niemeyer.

Lorenz, Gunter (1999): Adjective Intensification: Learners versus Native Speakers. A Corpus Study of Argumentative Writing. Amsterdam: Rodopi.

Scott, Mike (1997): WordSmith Tools. Version 2. Oxford: Oxford University Press.

Sinclair, John (1992): Trust the Text. In: Davies, Martin/Ravelli, Louise (Hg.) (1992): Advances in Systemic Linguistics. S. 5-19. London: Pinter.

Stubbs, Michael (2001): Words and Phrases: corpus studies of lexical semantics. Oxford: Blackwell.

Terrell, Peter/Schnorr, Veronika/Morris, Wendy V.A./Breitsprecher, Roland (1997): Collins-Pons German-English, English-German Dictionary. 3. neu bearbeitete Auflage. Glasgow: HarperCollins.

Wahrig, Gerhard (2000): Wahrig Deutsches Wörterbuch. 7. neu bearbeitete Auflage. 2000. Gütersloh/München: Bertelsmann Lexikon Verlag.

Woolls, David (1997): Multiconcord. Version 1.5. Birmingham: CFL Software Development. 